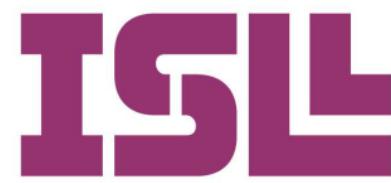

Número 5.

Enero de 2016

\title{
La lectura dialógica como medio para la mejora de la comprensión lectora
}

\author{
Raúl Gutiérrez Fresneda
}

Universidad de Alicante

Pág. 52 a la 58

\begin{abstract}
:
In our society, written The results weigh the potential value communication has a special of the program and support the significance, being an essential development of teaching models that reading for access to information integrate dialogic reading practices in and for school success. Children classroom dynamics as it facilitates acquire learning to read from the earliest ages from the relationship the learning of reading comprehension.
\end{abstract} with the people around them.

Dialogic learning is in the context of theories that emphasize interaction and dialogue as sources of learning so that the meaning, understanding and learning are enhanced through interactions that are established through a text. The purpose of this study was to analyze whether through learning programs that integrate dialogic reading along with the usual practices focused on teaching reading improved reading skills are acquired. A quasi-experimental comparison group design with pretest and posttest measures was used. In the study 120 students participated aged between 8 and 9 years. 


\section{Justificación}

El interés por el aprendizaje de la lengua escrita ha suscitado numerosos estudios y propuestas de intervención dirigidas a mejorar la comprensión de la información escrita. Sin embargo, en la actualidad no existe acuerdo sobre cuáles son los conocimientos o habilidades que mejor contribuyen al aprendizaje de la capacidad de comprensión ni cuáles son las prácticas docentes más idóneas para la facilitación de esta habilidad lingüística (Llamazares, Alonso-Cortés y Sánchez, 2015).

En lo que sí que existe acuerdo es que para lograr un buen desarrollo de la capacidad comprensiva es preciso formar a los alumnos en el acceso al lenguaje escrito desde sus inicios (Gutiérrez y Díez, 2015) y dotarles de las estrategias que les permitirán convertirse en lectores activos y expertos (OCDE, 2013). Tradicionalmente se ha equiparado la decodificación eficiente con un buen desempeño de la competencia lectora, asumiendo que la primera asegura la comprensión; pero lejos de esta realidad las investigaciones de las últimas décadas han modificado la concepción del término de lectura, identificándose actualmente tres teorías que lo explican. La primera concibe la lectura como un conjunto de habilidades orientadas a la mera transferencia de información, siendo necesario el dominio de tres facetas: la destreza para comprender lo que dice explícitamente el texto; la inferencia o habilidad para comprender la información que está implícita; y, la habilidad para evaluar la calidad del texto, las ideas y el propósito del autor. De acuerdo con esta concepción, el sentido del texto está en las palabras y oraciones que lo componen y el papel del lector es descubrirlo, de manera que el lector comprende el texto cuando es capaz de extraer el significado del mismo. Desde una nueva teoría, la lectura es concebida como el producto de la interacción entre el pensamiento y el lenguaje, se considera que entre el lector y el texto se establece una interacción. Desde esta perspectiva, la comprensión se entiende como un proceso en el que el lector utiliza las claves proporcionadas por el autor en función de su propio conocimiento o experiencia para inferir el significado que éste pretende comunicar. Es decir, los lectores no son sujetos pasivos frente a lo que leen, sino que emplean sus conocimientos previos interactuando con el texto, lo que les permite construir nuevos significados. Desde este postulado el lector alcanza la comprensión de un texto mediante la interacción de tres modelos: ascendente, descendente e interactivo. El modelo ascendente considera la lectura como un proceso secuencial y jerárquico que parte de la grafía y asciende hacia las unidades mayores (sílaba, palabra, frase o texto). Desde esta perspectiva se concede especial importancia al texto y la comprensión se concibe como un proceso de decodificación y reconstrucción del significado que se limita al resultado, sin atender al proceso. En el modelo descendente los conocimientos y experiencias del lector priman sobre el mensaje o el texto para acceder a la comprensión de la información.

Según este modelo las aportaciones que el lector transmite al texto tienen mayor importancia para su comprensión que lo que el texto dice. El modelo interactivo reconcilia los dos modelos anteriores y considera que ambos actúan de forma coordinada y simultánea. Entiende el proceso lector como una interacción entre el texto aportando su contenido y el lector activando sus conocimientos. A partir de la perspectiva interactiva surge el término de

ISL, vol. 5, 2016, págs. 52-58 ISNN: 2340-8685
Gutiérrez Fresneda, R (2016): La lectura dialógica como medio para la mejora de la comprensión lectora, Investigaciones Sobre Lectura, 5, 52-58. 
transacción, con la intención de reflejar que el significado deja de residir en el lector o en el texto, sino que se encuentra en la transacción entre ellos. Finalmente, desde esta nueva teoría, el significado que se construye es mayor que la suma de la información presente en el texto y las experiencias previas del lector, de forma que las características y los conocimientos previos del lector son tan importantes para la comprensión como las propias características del texto. De modo que, cuanto mayores sean los conocimientos previos de que disponga el lector, mayor será su capacidad para predecir y elaborar inferencias durante la lectura y para construir modelos adecuados del significado del texto. Esta teoría se diferencia de la anterior en que el significado que se crea cuando el lector y el autor se encuentran en los textos es superior al que ofrece el texto y a los conocimientos que tenía el lector. Es decir, el significado que cada lector puede elaborar a partir de un mismo texto no tiene por qué coincidir exactamente con el de los demás, pues dependerá de las transacciones que se produzcan entre los lectores y los textos en un contexto específico.

Leer es un proceso cognitivo complejo que precisa la utilización de estrategias de alto nivel: dotarse de objetivos, establecer y verificar predicciones, controlar lo que se va leyendo, tomar decisiones respecto a las dificultades o carencias de comprensión que se van produciendo, identificar la información principal, lo que requiere la implicación activa del lector.

En consecuencia, la comprensión como proceso de construcción de significados requiere que los procesos de enseñanza se centren en la transacción del lector con el texto, lo que demanda de un lector dialógico, que interactúe con la información del texto poniéndose en diálogo con el autor, formulando preguntas, hipótesis, haciendo inferencias, adoptando un planteamiento crítico.

Sin embargo, la investigación en torno a la comprensión escrita se ha centrado en la relación entre el lector y el texto como un proceso individual desestimando el potencial que puede alcanzarse si el acto lector se torna colectivo (Agustí, Ballart y García, 2014).

La comprensión lectora es un proceso complejo donde el lector participa de manera activa poniendo en juego una serie de estrategias y conocimientos que le permiten interaccionar con los significados del texto, lo que de hacerse de manera conjunta entre varias personas verbalizando y poniendo en común sus propias estrategias lectoras puede contribuir en gran medida al logro de los mecanismos necesarios para un aprendizaje lector más eficaz.

El objetivo de este trabajo, fue analizar el efecto que la intervención de un programa centrado en el desarrollo de las estrategias de compresión lectora basado en la lectura dialógica presenta en la mejora de la comprensión lectora. Para ello, se compara el grado de adquisición de la comprensión lectora en dos muestras de alumnos de entre 8 y 9 años, uno que recibe intervención en el proceso de comprensión lectora mediante la instrucción en 
estrategias de lectura en grupos interactivos, y otro que sigue el mismo programa de enseñanza de estrategias lectoras, pero a través de la clase magistral tradicional. Nuestra hipótesis es que el alumnado perteneciente al grupo en el que sus integrantes verbalizan y ponen en común las estrategias que se establecen a través de un texto, obtendrá un mejor rendimiento en el desarrollo de la comprensión lectora.

\section{Método}

\subsection{Participantes}

En el estudio participaron 120 alumnos con edades comprendidas entre los 8 y los 9 años $($ Media $=8,23$; DT $=0,41)$, de los cuales el 49,3\% eran niños y el 50,7\% niñas. Estos alumnos pertenecían a dos centros educativos de la provincia de Alicante, que compartían la característica de estar ubicados en un contexto sociocultural de nivel medio.

\subsection{Diseño y procedimiento}

El estudio utilizó un diseño cuasiexperimental de medidas repetidas pretestpostest con grupo control. Antes y después de implementar el programa de intervención se aplicó una batería de dos instrumentos de evaluación a los participantes experimentales y control con la finalidad de medir la variable dependiente sobre la que se hipotetizó que el programa iba a tener efecto la comprensión lectora. La aplicación de la batería de pruebas antes y después de aplicar el programa se llevó a cabo por profesionales de la educación (maestros especialistas en Audición y Lenguaje y psicopedagogos).

\subsection{Instrumentos}

Para la recogida de información sobre el grado de dominio del proceso lector se emplearon las siguientes pruebas:

- Test ACL-3 (Catalá, Catalá, Molina y Monclús, 2001). Se compone de siete textos con 25 ítems de diferente tipología textual: narrativa, expositiva y retórica. Valora la comprensión lectora mediante la respuesta a preguntas de carácter literal, inferencial, de reorganización de la información y de valoración crítica. Se concede un punto por cada respuesta correcta. La fiabilidad medida con el coeficiente KR-20 es de 0.80 .

- Escala de conciencia lectora (ESCOLA) (Puente, Jiménez y Alvarado, 2009). Es un cuestionario para la evaluación de las habilidades metacognitivas relacionadas con la lectura. Los ítems evalúan tres procesos metacognitivos: planificación lectora, supervisión y evaluación. Se compone de 56 ítems, cada ítem presenta tres opciones de respuesta, las cuales se puntúan con 0,1 ó 2 . La consistencia interna de la escala calculada mediante el alfa de Cronbach presenta un valor de 0.85 


\section{Resultados}

En la Tabla 1 y 2 se muestran los datos obtenidos por el grupo control y experimental en el pretest en las pruebas ACL-3 y ESCOLA en de cada uno de los niveles y estrategias de comprensión lectora, de igual modo en las Tablas 3 y 4 se presentan los resultados obtenidos en esas mismas pruebas en el postest. En las tablas 5 y 6 se ofrecen los datos referentes al grado de comprensión lectora respecto del género del alumnado.

Tabla 1: Resultados obtenidos por el grupo control y experimental en el pretest en la prueba $A C L-3$

\begin{tabular}{lcccc}
\hline & C. Literal & C. Inferencial & C. Reorganizativa & C. Crítica \\
Grupo Control & .251 & .233 & .158 & .465 \\
Grupo Experimental & .265 & .446 & .321 & .224 \\
\hline Nota. ${ }^{*} \mathrm{p}<, 05 ; * * \mathrm{p}<, 01 ; * * * \mathrm{p}<, 001$ & & &
\end{tabular}

Tabla 2: Resultados obtenidos por el grupo control y experimental en el pretest en la prueba ESCOLA

\begin{tabular}{lccc}
\hline & Planificación & Supervisión & Evaluación \\
Grupo Control & .342 & .423 & .253 \\
Grupo Experimental & .146 & .212 & .241 \\
\hline Nota. ${ }^{*} \mathrm{p}<, 05 ; * * \mathrm{p}<, 01 ; * * * \mathrm{p}<, 001$ & &
\end{tabular}

Tabla 3: Resultados obtenidos por el grupo control y experimental en el postest en la prueba $A C L-3$

\begin{tabular}{lcccc}
\hline & C. Literal & C. Inferencial & C. Reorganizativa & C. Crítica \\
Grupo Control & .224 & $.423^{*}$ & $.346^{*}$ & .284 \\
Grupo experimental & .327 & $.564 * *$ & $.642^{* *}$ & $.478^{* * *}$ \\
\hline Nota. ${ }^{*} \mathrm{p}<, 05 ; * \mathrm{p}<, 01 ; * * \mathrm{p}<, 001$ & & &
\end{tabular}

Tabla 4: Resultados obtenidos por el grupo control y experimental en el postest en la prueba ESCOLA

\begin{tabular}{lccc}
\hline & Planificación & Supervisión & Evaluación \\
Grupo Control & .225 & .321 & .475 \\
Grupo Experimental & $.342 * *$ & $.352 * *$ & $.356^{* *}$ \\
\hline Nota. ${ }^{*} \mathrm{p}<, 05 ; * * \mathrm{p}<, 01 ; * * * \mathrm{p}<, 001$ & &
\end{tabular}


Como se puede observar en la Tablas 1,2 no existen diferencias significativas entre los grupos control y experimental antes de iniciar el programa de intervención ni en los distintos tipos de comprensión ni en las estrategias lectoras. Sin embargo, sí que se observan diferencias en las variables comprensión inferencial, reorganizativa y crítica a favor del grupo que ha participado en el programa de intervención, así como en las tres estrategias de comprensión: planificación, supervisión y evaluación.

Tabla 5: Resultados obtenidos por género en el postest en la prueba ACL-3

\begin{tabular}{lcccc}
\hline & C. Literal & C. Inferencial & C. Reorganizativa & C. Crítica \\
niños & .125 & .143 & .442 & .152 \\
niñas & .223 & .267 & .342 & .346 \\
\hline Nota. ${ }^{*} \mathrm{p}<, 05 ; * * \mathrm{p}<, 01 ; * * * \mathrm{p}<, 001$ & &
\end{tabular}

Tabla 6: Resultados obtenidos por género en el postest en la prueba ESCOLA

\begin{tabular}{lccc}
\hline & Planificación & Supervisión & Evaluación \\
niños & .312 & .125 & .272 \\
niñas & .215 & .257 & .152 \\
\hline Nota. $* \mathrm{p}<, 05 ; * * \mathrm{p}<, 01 ; * * * \mathrm{p}<, 001$ &
\end{tabular}

En las Tablas 5 y 6 observamos que tanto en el caso de los niños como de las niñas los resultados son muy similares en las diferentes variables estudiadas, por lo que se comprueba que no se produjeron efectos diferenciales del programa en función del género.

\section{Discusión}

El objetivo de este estudio era comprobar el efecto que la intervención de un programa orientado al desarrollo de las estrategias de compresión basado en la lectura dialógica presenta en la mejora de la competencia lectora. Los resultados obtenidos ponen de manifiesto que la instrucción a través de grupos interactivos mejora de manera muy significativa el proceso de adquisición de la lectura. En línea con otros aportes coincidimos con Gutiérrez-Braojos y Salmerón (2012) cuando señalan que los escolares pueden experimentar una mejora en el uso de estrategias si se implementan experiencias de aprendizaje adecuadas.

En definitiva, la lectura dialógica constituye un medio muy útil tal y como hemos comprobado para la mejora de la comprensión, ya que posibilita un incremento de las interacciones alrededor de las actividades de lectura. El aprendizaje de las estrategias lectoras se adquiere por medio de las habilidades comunicativas que se llevan a cabo, de

ISL, vol. 5, 2016, págs. 52-58 ISNN: 2340-8685
Gutiérrez Fresneda, R (2016): La lectura dialógica como medio para la mejora de la comprensión lectora, Investigaciones Sobre Lectura, 5, 52-58. 
los conocimientos previos que poseen los distintos compañeros sobre la información textual, de las aportaciones personales y de la reflexión que se va efectuando sobre cada una de las estrategias implementadas sobre el proceso de lectura, lo que favorece la toma de decisiones sobre qué estrategias de lectura aplicar, cómo aplicarlas de manera eficaz, cuándo aplicar cada una de ellas,... lo que permitirá al lector, identificar, seleccionar y utilizar las estrategias más adecuadas ante las distintas situaciones y tipologías textuales.

A partir de los resultados de este trabajo, a nivel práctico se sugiere el diseño de programas orientados al desarrollo de las estrategias lectoras, en concreto hacia aquellas que contribuyan a la activación de conocimientos previos, a la identificación de ideas principales, la construcción de inferencias, el uso de organizadores gráficos, y a la supervisión del proceso de comprensión, a través de la configuración de grupos interactivos que potencien la puesta en común de las estrategias lectoras.

De modo que, hemos dado respuesta a una de las cuestiones que se vienen demandando desde el ámbito científico en los últimos años, la cual hace referencia a la necesidad de analizar qué aportaciones efectúa el entrenamiento de las estrategias lectoras al aprendizaje de la competencia lectora en nuestra lengua.

\section{Bibliografía:}

Agustí, M., Ballart, M. y García, M. (2014). Aprender a leer con la lectura compartida. Otra lectura es posible. Aula de innovación educativa, 231, 39-43.

Catalá, G., Catalá, M., Molina, E. y Monclús, R. (2001). Evaluación de la comprensión lectora. Barcelona: Graó.

Gutiérrez, R. y Díez, A. (2015). Aprendizaje de la escritura y habilidades de conciencia fonológica en las primeras edades. Bordón, 67(4), 43-59.

Gutiérrez-Braojos y Salmerón, H. (2012). Estrategias de comprensión lectora: enseñanza y evaluación en educación primaria. Revista profesorado, 16(1), 183-202.

Llamazares M. T., Alonso-Cortés, M. D. y Susana S. (2015). Factores que influyen en el aprendizaje de la comprensión lectora y de la composición escrita: tres estudios realizados en aulas de Educación Infantil y Educación Primaria. Investigaciones sobre Lectura, 3, 67-82

OECD (2013). PISA 2012 Results: What students know and can do. Student performance in mathematics, reading and science (Vol. 1). Recuperado de: http://www.oecd.org/pisa/keyfindings/pisa-2012-results.htm 
Puente, A. Jiménez, V. y Alvarado, J. M. (2009). Escala de conciencia lectora (ESCOLA). Evaluación e intervención psicoeducativa de procesos y variables metacognitivas durante la lectura. Madrid: EOS.

ISL, vol. 5, 2016, págs. 52-58 ISNN: 2340-8685
Gutiérrez Fresneda, R (2016): La lectura dialógica como medio para la mejora de la comprensión lectora, Investigaciones Sobre Lectura, 5, 52-58. 